\title{
Is Animal-based Biomedical Research Being Used in Its Original Context?
}

\author{
Constança Carvalho \\ Centre for Philosophy of Sciences of the University of Lisbon, Portugal \\ constanca.carvalho@sapo.pt
}

\begin{abstract}
Daniel Alves
Department of Animal Biology, Centre for Ecology Evolution and Environmental Changes, Portugal Faculty of Sciences of the University of Lisbon
\end{abstract}

Andrew Knight

Centre for Animal Welfare, University of Winchester, U K

Luis Vicente

Centre for Philosophy of Sciences of the University of Lisbon, Portugal

\section{Introduction}

Since the second half of the twentieth century, non-human animals (hereinafter referred to as animals) have been widely used as models for researching human disorders. Historically, this occurred for two main reasons: a) animals are complex living systems; and b) it is considered less ethically-contentious as well as easier, quicker, and cheaper to use animals than humans. Their benefit for biomedical advancement is assumed even though systematic evaluations, though uncommon, suggest otherwise. It is crucial to evaluate whether animal-based biomedical research successfully benefits medical researcheven through indirect pathways — or if it is being used merely to justify further animal-based research. In this chapter we demonstrate that there is a lack of communication between animal-based research and clinical research. We discuss possible reasons for this and reflect on whether animal use in biomedical research is, indeed, fulfilling its primary purpose.

Humans share a long evolutionary story with the rest of the animal kingdom, which explains common physiological and behavioral traits and adaptations. For example, basal ganglia, a set of subcortical nuclei involved in

(C) CONSTANÇA CARVALHO ET AL., 2019 | DOI:10.1163/9789004391192_017

This is an open access chapter distributed under the terms of the prevailing CC-BY-NC License at the time 9004391192 of publication. 
several motor functions, are present throughout vertebrate taxa and are largely similar across species (Lee et al., 2015). Similarly, the rise of body temperature as a response to infection is shared by humans and other mammals (Nesse and Williams, 1996; Schaffner, 2006). Even poikilothermic (cold-blooded) animals, such as lizards, tend to move to warmer places when they are ill, until their body temperature is several degrees above normal (Nesse and Williams, 1996). The relatively recent decoding of genomes had shown an impressive number of genes shared between ourselves and taxonomically-distant species, such as the frog (Hellsten et al., 2010). These similarities provided the basis for the untested assumption that animals provide good research models for human disorders.

However, we know that minimal biological changes can create significant differences between species and individuals. For example, Darwin's finches comprise 14 closely-related species that vary dramatically in their feeding habits, despite their biological proximity (Lack, 1947). Even amongst individuals of the same species, slight and almost undetectable differences can cause very different adaptive responses. For example, human beings with sickle cell trait may have increased protection from malaria but risk sudden death by hypoxia, when visiting high altitudes or performing intense physical exertion (Scheinin and Wetli, 2009; Webber et al., 2016), safe activities for most people.

Despite individual differences, it is obvious that human beings are the best biomedical model for human disorders. However, clinical research is time consuming and can have severe ethical constraints, which is one of the main reasons why animals are widely used as models for human disorders. Recent in vitro developments have allowed us to create cultures of human cells and tissues (e.g., Petropolis et al,, 2016; Wilson, Ahearne and Hopkinson, 2015) that are considered superior to using animal samples for human-based research (Clemedson et al., 1998; Huhtala et al., 2008; Petropolis et al., 2016). Nonetheless, among the scientific community, the main obstacle to the total replacement of animal use in biomedical research is not a desire to study cells, tissues, or organs, but the desire to study entire, functioning bodily systems. This is considered necessary when objectives include understanding a drug effect in the whole organism or trying to understand the etiology and pathogenesis of multifactorial disorders, such as mental disorders.

In silico techniques have been slowly addressing this issue, creating whole body simulations (e.g., Viceconti, Clapworthy and Jan, 2008; Viceconti, Henney and Morley-Fletcher, 2016). However, the availability of human data limits these models. For example, if a new disease arises, models may fail to predict accurately the response of the human body to the new pathogen due to lack of data. It should be noted that animal models also suffer from failure to predict human 
responses accurately. Despite the accepted potential of in silico techniques, unvalidated animal models are still commonly believed to be the best available, so far, for studying the entire, functioning human body.

Throughout the years, various authors have asserted that animal research has made only poor contributions to medical progress (e.g., Bailey, 2008; Fadali, 1996; Greek and Greek, 2003; Shapiro, 1998), while others have asserted the opposite (e.g., Illman, 2008; Shively and Clarkson, 2009; Perretta, 2009). Such assertions are based upon historical analyses, investigations into the development of various treatments, and critical reviews of animal model use. Historical accounts are disputed. A classic example is the discovery of the role of the pancreas in diabetes. Many claim that we owe this discovery to experiments conducted by Minkowski and von Mering with dogs, in the second half of the nineteenth century (cited in Bliss, 1982); whereas, others argue that this medical breakthrough was achieved by Thomas Cawley, 100 years earlier, while performing autopsies on patients who died from diabetes (cited in Fadali, 1996).

Investigations into the development of treatments are also controversial. A good example is the development of the poliomyelitis vaccine. Poliomyelitis is a viral disease that reached epidemic proportions in 1916. Some (e.g., Illman, 2008) state that it was the experiments performed on mice and monkeys that allowed scientists to understand its pathogenesis and develop a vaccine. Furthermore, both poliomyelitis vaccines (Salk vaccine and Sabin vaccine) were initially grown in monkey kidney tissue (Dowdle et al., 2003), reinforcing the perception of the central role of animal experiments in the development of poliomyelitis treatment (Illman, 2008). However, others (e.g., Fadali, 1996) claim that animal experiments delayed the vaccine's development. Rhesus monkeys, which provided a widely-used animal model for poliomyelitis, misled scientists to believe that the virus was transmitted via the respiratory, rather than the digestive route (Dowling, cited in Bailey, 2008), as earlier research on humans had suggested (see Fadali, 1996, for a review). This mistake led to an erroneous clinical trial in 1937, in which exposed children suffered olfactory damage (Parish, 1968). Also, the first poliomyelitis vaccines, grown on monkey kidney cells, were responsible for the exposure of millions of American citizens to simian virus 40, found in rare human cancers (Pennisi, 1997). When it comes to non-human primates (NHPS), these disputes are even more contentious, because public opinion is less supportive of the use of NHPs in research (European Commission, 2010). Furthermore, as technology evolves, better methods become available, and the apparent historical necessity of animal experiments becomes of less relevance. For example, vaccines that used to be developed using animal tissues—at times suboptimally due to poor efficiency (e.g., rubella 
vaccine developed through duck embryo cells and dog kidney cells) or zoonosis (e.g., the simian virus that reached humans through the first polio vaccines) - are now being developed using human strains (Plotkin, 2017).

Recently more objective tools to assess the contribution of animal models to biomedical progress have emerged. Such is the case of systematic reviews, meta-analyses, and citation analyses. Systematic reviews are literature reviews focused on a research question that aim to identify, appraise, and synthesize all high-quality research evidence relevant to that question. They are generally considered the best tool to produce evidence about the value of animal studies (Pound et al., 2004), not only because they are designed to include all relevant information, drastically reducing the potential for bias; butalso because systematic reviews evaluate experimental designs through rigorous and objective peerreviewed protocols, such as the Animal Research:Reporting In Vivo Experiments (ARRIVE) guidelines that apply scientific method to the task of reviewing research evidence (Kilkenny et al., 2010). A meta-analysis can go even further by incorporating a statistical representation of all the reviewed studies as well.

In the past decades, the number of systematic reviews shedding light on the scientific value of animal studies has increased (e.g., Banwell, Sena and Macleod, 2009; Corpet and Pierre, 2005; Lucas et al., 2002; Macleod et al., 2005; Martić-Kehl et al., 2015; Perel et al., 2007). The systematic reviews have revealed: a) poor transferability of animal outcomes to human clinical trials (e.g., Perel et al., 2007); b) simultaneous occurrence of animal and clinical trials, rather than sequentially, as expected given that the animal experiments should be conducted first, to allow detection of possible toxicity (e.g., Lucas et al., 2002); and, c) significant methodological and design flaws in a clear majority of animal experiments (e.g., Martić-Kehl et al., 2015). Consequently, the use of ARRIVE or similar guidelines has become more common, which will hopefully lead to better protocols and reduce redundant studies. As for the poor transferability of animal outcomes to human trials, it can be argued that this is either a consequence of poor experimental design, and/or the fact that animal models are not suitable models for human beings (Bailey and Taylor, 2016).

Another way to determine the value of animal studies is citation analysis, which consists of determining the frequency with which a study is cited in subsequent papers. Several authors have conducted citation analyses on published papers, reporting data from animals used as models for human disorders (e.g., Carvalho et al., 2016; Knight, 2007; Long, Huang and Ho, 2014); results show that these papers have received very few citations in human medical papers. Again, it can be debated whether this occurs due to a false assumption that animal models are suitable models for human disorders or because of methodological errors, or both. 
To try to address this issue, we performed a citation analysis on a small sample of papers reporting data from animals used to model two complex psychiatric disorders: attention deficit hyperactivity disorder (ADHD) and major depressive disorder (MDD).

ADHD is a chronic neurodevelopmental condition of multifactorial origin, marked by persistent inattention; hyperactivity; and, occasionally, impulsivity (American Psychiatric Association, APA, 2013). It affects $2.2 \%$ of children worldwide (Erskine et al., 2013); and it can be extremely disabling (APA, 2013). MDD is a complex psychiatric mood disorder characterized by a persistent feeling of sadness that seriously impairs normal day-to-day functioning and may even lead to suicide (APA, 2013). Mental disorders are the leading cause of years lived with disability worldwide, and $40.5 \%$ of this burden is caused by MDD alone (World Health Organization, 2008).

In this study we categorized the citations obtained into animal versus human studies and determined whether human-based and animal-based papers focused on the same disorder investigated by the animal study they were citing. This form of analysis is valuable for shedding light on whether animal-based research is being used to advance human healthcare, or whether it simply fuels further animal-based research. If animal studies are contributing to human healthcare advancements, then we would expect that:

1. The citations made in human-based papers should be a substantial proportion of total citations.

2. The citations should be made mainly by studies focused on the same disorder. Any substantial deviations would signal the possibility that animal-based research is not achieving its primary purpose.

\section{Methods}

We conducted a citation analysis as defined by Garfield and Merton (1979). Briefly, in a citation analysis, one defines a number of target papers and conducts a search of all papers that cite these target papers. The information obtained can include the total number of citations and patterns of citation. We used a total of 50 target papers: 25 non-human animal studies on ADHD, and 25 non-human animal studies on MDD.

The ADHD papers were selected from the citation analysis database created in the study by Carvalho et al. (2016). We included all papers reporting data collected with primate models (7 papers) and randomly selected 18 papers from the remaining papers, using the free online tool, Research Randomizer (www.randomizer.org). The 25 studies were examined to determine the proportion of citations each paper received in human-based papers focused 
on ADHD, in human-based papers focused on other subjects, in animalbased papers focused on ADHD, and in animal-based papers focused on other subjects.

The MDD papers were obtained using PubMed to locate original articles using animal models to investigate major depressive disorder (similar to the protocol used in Carvalho et al., 2016). We searched PubMed using the following Medical Subject Heading (MeSH) search terms:

\begin{abstract}
"Major Depressive Disorder" AND (title/abstract): "animal" or "rat" or "mice" or "mouse" or "Rattus" or "Mus" or "pig" or "Cavia" or "Sus" OR "rabbit" OR "Leporidae" OR "Drosophila" or "primate" or "monkey" or "Macaca" or "macaque" or "ape" or "rhesus" or "chimpanzee" or "bonobo" or "gorilla" oR "Pan" OR "Orang Utan" oR "Pongo" OR "gibbon" OR "Hylobates" OR "Colobus" OR "Baboon" OR "Papio" OR "Mandrillus" OR "Mandrill" on "Cebus" or "Cebuella" on "Brachyteles" or "Loris" on "Nycticebus" or "Lemur" on "dog” or "Canis" or "cat" or "Felis."
\end{abstract}

We found 33 published papers using NHPs as models and randomly selected seven, using the same randomizing tool. We found over 1,ooo published papers using other animals as models and proceeded, as above, to randomly select 18 papers for the citation analysis. We recorded the number of citations each paper received from subsequent animal research papers and subsequent human research papers. We similarly analyzed the aim of the citing paper (whether it was focused on the same disorder or another), in both animal and human papers.

Using Fisher's exact test (http://www.kisnet.or.jp/nappa/software/star-e/ freq/1x2.htm), we investigated whether there was a significant difference between the number of citations of the target animal articles in human research papers and in animal research papers. We also verified whether there was a significant difference between the number of citations in subsequent articles addressing the same disorder and subsequent articles addressing different topics. Differences were considered statistically significant if $p<0.05$.

\title{
$3 \quad$ Results
}

Regarding our ADHD sample, the 25 original animal studies were cited 660 times. As shown in Figure 16.1, animal studies were mainly cited in other animal research papers (315), of which 82 focused on ADHD and 233 focused on different subjects. The sample resulted in 69 citations in human research papers, of which 30 focused on ADHD and 39 focused on different subjects. The 


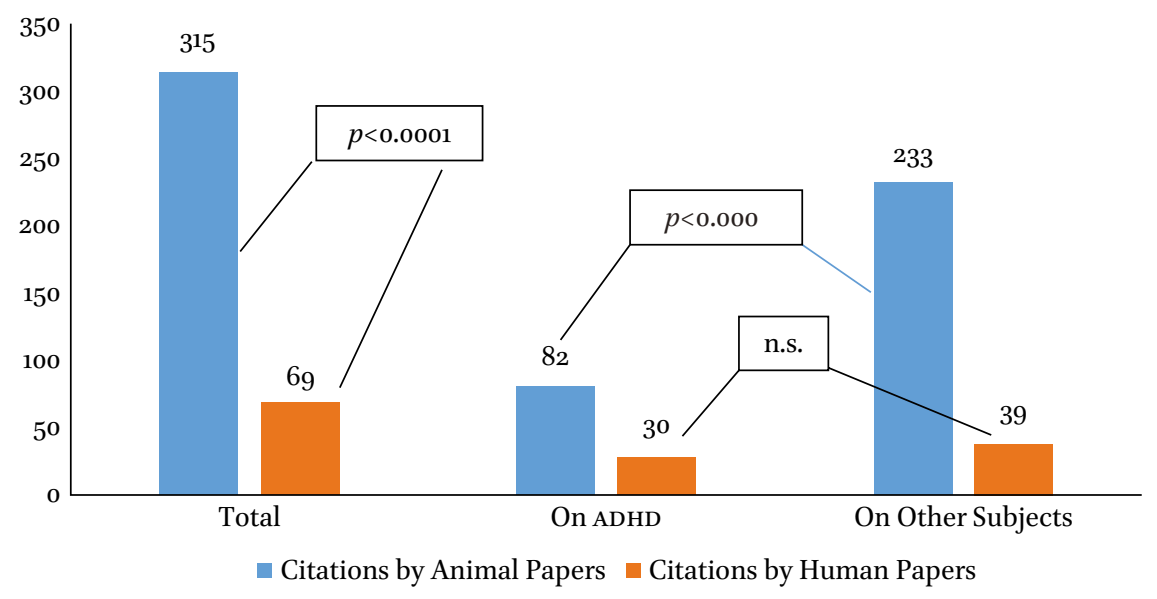

FIGURE 16.1 Citations of animal papers focused on ADHD.

remaining 345 citations were in review articles (198) or papers describing different methods, such as in silico or in vitro (147).

The columns represent the number of citations of the 25 target papers in animal research papers (blue) and human research papers (orange). The total number (left), as well as the number of citations in papers studying ADHD (middle) and other subjects (right) are presented. Fisher's exact test $p$-values are also presented for each comparison made (n.s.= non-significant).

The number of citations in animal research papers was far greater than the number of citations in human research papers $(p<0.0001)$. The number of citations in animal research papers focused on ADHD was lower than the number of citations in animal research papers focused on other subjects $(p<0.0001)$. The difference between the number of citations in human research papers on ADHD and human research papers focused on other subjects was not statistically significant $(p=0.3355)$.

Seven of the target papers reported NHP studies. These papers received 274 citations, 94 of which were in subsequent animal research papers and 48 were in human research papers. The remaining 138 citations were in review papers (96) or papers describing different methods, such as in silico or in vitro (42). The difference between citations in animal research papers and human research papers was statistically significant $(p=0.0001)$. Of the 94 citations in subsequent animal papers, 21 were in papers focused on $\mathrm{AD} H \mathrm{D}$, and 73 were in papers focused on other issues. This difference was also statistically significant $(p<0.0001)$.

Of the 48 citations in human research papers, 15 were in papers focused on ADHD, and 33 were in papers describing other disorders. Fisher's exact test showed that in the case of NHPs there was a statistically significant difference 


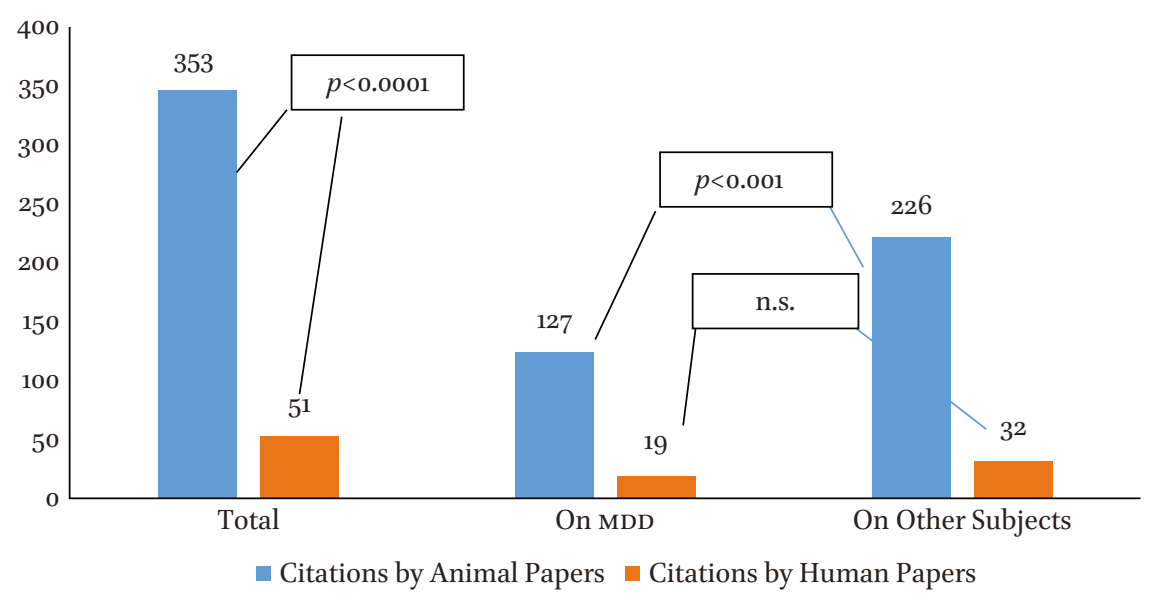

FIGURE 16.2 Citations of animal papers focused on MDD.

between the number of citations in papers on ADHD and papers focused on other subjects $(p=0.0132)$.

Regarding the MDD sample, the 25 target animal studies were cited 631 times. As shown in Figure 16.2, animal studies were mainly cited in other animal research papers (353), of which 127 focused on MDD, and 226 focused on different subjects. The sample received $5^{1}$ citations in human research papers, of which 19 focused on MDD, and 32 focused on different subjects. The remaining 227 citations were in review articles (163) or papers describing different methods, such as in silico or in vitro (64).

The columns represent the number of citations in animal research papers (blue) and in human research papers (orange) of the 25 cited papers. The total number (left), as well as the number of citations in papers studying MDD (middle) and other subjects (right) are presented. Fisher's exact test $p$-values are also presented for each comparison made.

The number of citations in animal research papers was substantially greater than the number of citations in human research papers (Fisher's exact test, $p<0.0001)$. The number of citations in animal research papers focused on MDD was lower than the number of citations in papers focused other subjects $(p<0.0001)$. The difference between the number of citations in human research papers focused on MDD and papers focused on other subjects was not statistically significant $(p=0.0919)$.

The seven papers reporting on NHP studies received 227 citations, 97 of which were in subsequent animal research papers, and 19 were in human research papers. This difference was statically significant $(p=0.001)$. Of the 97 
citations in subsequent animal papers, 13 were in papers on MDD, and 84 were in papers focused on other issues. This difference was statistically significant $(p<0.0001)$. Of the 19 citations in human medical papers, six were in papers on MDD, and 13 were in papers focused on other subjects. This difference was not statistically significant $(p=0.1670)$.

\section{4 \\ Discussion}

Our results suggest that animal data is mainly used by subsequent animal papers. Another trend that emerged is that papers citing animal research (whether they focus on human medical research or not) focus on disorders that differ from the one targeted in the animal study cited. This trend is stronger in papers focused on animal research.

The tendency for animal research to be cited more in subsequent animal research has been previously described (e.g., Carvalho et al., 2016). This finding contradicts the previously stated assumption that citations in human-focused papers should constitute a substantial proportion of the total number of citations. Clearly, biomedical research focused on animal models does not seem to be considered important by, or particularly visible to, the human medical research community.

Our results also indicate that papers citing data collected from animal models do not necessarily target the disorder described in the animal paper. This difference appears to be more significant in animal research papers citing other animal research papers, than in human research papers that cite animal research. This contradicts the second assumption we tested: that citations should be made mainly by studies focused on the same disorder. This finding reinforces the concern that animal-based research is failing to shape meaningful healthcare advances for humans.

It can be argued that if the same animal model is used for different disorders, it may contribute more to medical research than predicted by the second assumption. For example, DAT knock-out mice comprise a common model for ADHD but are also used to model Parkinson or schizophrenia (Gainetdinov, 2008). Nevertheless, the total citation frequency in human research papers is still very low, regardless of the paper's area of focus (Carvalho et al., 2016; Knight, 2007; Long, Huang and Ho, 2014).

The fact that animal strains are used to model several disorders may help explain the intriguing tendency for animal research papers to be cited more often in papers addressing non-related subjects than in papers focused on the same disorder. This tendency was also apparent in human-based papers that cited animal-based papers focused on MDD. This may have occurred because 
there are 6-7 times more papers focused on MDD than on ADHD, which may mean that the 25 papers on $M D D$ were not a representative sample of $M D D$ research. If this phenomenon was to recur with a larger sample, one could argue that this is due to the same animal strain being used for different purposes, as previously mentioned. If the strains used in MDD research are commonly used to model a greater number of disorders than strains used in ADHD research, it would be more probable that human studies focused on unrelated disorders cite studies in these strains. We did not verify this, and it should be explored in future studies.

Our data shows that even though the difference between the total frequency of citations by human papers focused on ADHD and paper focused on other subjects was not statistically significant, there was a bias regarding papers describing NHP models of ADHD. A close examination of the data allowed us to conclude that this bias was due to one paper, cited 18 times in human research papers, 17 of which focused on disorders other than ADHD. This particular paper described the behavioral changes caused by bicuculline microinjections in external globus pallidus, a brain structure involved in pathogenesis of ADHD but also in Tourette's syndrome. Most of the 18 citations this paper received in human papers were actually from papers related to Tourette's syndrome. If we discard this outlier, the data on NHP follows the same pattern as other ADHD papers.

Since our two assumptions have been challenged, we must discuss their causes and implications. One possible explanation for these results is that animal models only attempt to model specific symptoms or traits of complex human disorders. This oversimplification may lead to results that are non-applicable or of minimal use for human medicine. Another possible explanation is that funding is more easily attached to studies that claim to have the potential to advance human health. This may lead animal researchers to overestimate the applicability of their projects. A further possible explanation is that communication and sharing of ideas between clinical and preclinical research is insufficient. Moreover, previous studies have shown that clinical and preclinical trials can occur simultaneously (Pound et al., 2004), which emphasizes this lack of communication. Although it is difficult to define an optimum communication level, this issue must be raised in both communities in order to maximize efficiency in scientific research as well as the promotion of animal welfare. An additional possibility is that a substantial amount of animal research is needed in order to achieve a critical mass that can lead to useful breakthroughs in human health. This is a theoretical possibility that is difficult to measure and properly test. However, even if proven correct, the financial and ethical implications of this assumption should be considered. Other methods may prove to be more efficient or ethically acceptable, and this 
comparison could lead to a reevaluation of funding priorities. Finally, a conceivable possibility is that animal models are not suitable for biomedical research into complex human disorders. It may be possible that the uniqueness of some human disorders is just not feasibly simulated in non-human animals.

If our last suggested explanation is indeed correct, the implications must be considered. The funding currently allocated to these animal-based studies should still be available for science. While most of it would likely be redirected to other models of these disorders, some of it could be assigned either to other basic research fields or to the care of surplus animals.

Regardless of the possible explanations, our results indicate that animalbased research is failing to reach the human medical community, at least in the case of mental disorders, such as the ones we evaluated. This means that considerable financial investment and considerable suffering inflicted on the animals used for this purpose did not translate into direct medical advances. It would be interesting to survey the practitioners working with mental disorders to assess if this is due to lack of awareness of animal-based findings, or if they consider animal-based data to be inadequate or lacking in relevance.

In conclusion, our analysis suggests that most animal-based research, at least in the case of these mental disorders, is not currently being utilized by human-based researchers. Regardless of the reasons for this, the profound financial and ethical implications should lead to a reevaluation of the current research paradigm, which is heavily reliant on invasive animal use.

\section{Acknowledgments}

This study was financed by Animalfree Research - Switzerland and Portuguese National Funds through Centre for Philosophy of Sciences of the University of Lisbon (CFCUL) Unit funding UID/FIL/00678/2013.

\section{References}

American Psychiatric Association (APA) (2013). Diagnostic and statistical manual of mental disorders. 5th ed. Arlington, va: American Psychiatric Publishing.

Bailey, J. (2008). An Assessment of the Role of Chimpanzees in AIDS Vaccine Research. Alternatives to Laboratory Animals, 36(4), pp. 381-428.

Bailey, J. and K. Taylor (2016). Non-human Primates in Neuroscience Research: The Case Against Its Scientific Necessity. Alternatives to Laboratory Animals, 44(1), pp. 43-69. 
Banwell, V., E. Sena and M. Macleod (2009). Systematic Review and Stratified Metaanalysis of the Efficacy of Interleukin-1 Receptor Antagonist in Animal Models of Stroke. Journal of Stroke and Cerebrovascular Diseases, 18, pp. 269-276.

Bliss, M. (1982). The Discovery of insulin: 25th anniversary edition. Chicago, IL: Chicago University Press.

Carvalho, C., M.V. Crespo, L.F. Bastos, A. Knight and L. Vicente (2016). Contribution of Animal Models to Contemporary Understanding of Attention Deficit Hyperactivity Disorder. Alternatives to Animal Experimentation, 33(3), pp. 243-249.

Clemedson, C., M. Andersson, Y. Aoki, F.A. Barile, A.M. Bassi, M.C. Calleja, A. Castano, R.H. Clothier, P. Dierickx, B. Ekwall, M. Ferro, G. Fiskesjö, L. Garza-Ocanas, M.J. Gómez-Lechón, M. Gülden, T. Hall, K. Imai, B. Isomaa, A. Kahru, G. Kerszman, P. Kjellstrand, U. Kristen, M. Kunimoto, S. Kärenlampi, L. Lewan, H. Lilius, A. Loukianov, F. Monaco, T. Ohno, G. Persoone, L. Romert, T.W. Sawyer, H. Segner, H. Seibert, R. Shrivastava, M. Sjöström, A. Stammati, N. Tanaka, A. Thuvander, O. Torres-Alanis, M. Valentino, S. Wakuri, E. Walum, X.H. Wang, A. Wieslander, F. Zucco and B. Ekwall (1998). MEIC Evaluation of Acute Systemic Toxicity, Part IV. In Vitro Results from 67 Toxicity Assays Used to Test Reference Chemicals 31-50 and a Comparative Cytotoxicity Analysis. Alternatives to Laboratory Animals, 26, pp. 131-183.

Corpet, D. and F. Pierre (2005). How Good Are Rodent Models of Carcinogenesis in Predicting Efficacy in Humans? A Systematic Review And Meta-analysis of Colon Chemoprevention in Rats, Mice and men. European Journal of Cancer, 41, pp. 19111922.

Dowdle, W., D. Gourville, O. Kew, M. Pallansch and D. Wood (2003). Polio Eradication: The OPV Paradox. Reviews in Medical Virology, 13(5), pp. 277-291.

Erskine, H.E., A.J. Ferrari, P. Nelson, G.V. Polanczyk, A.D. Flaxman, T. Vos, H.A. Whiteford and J.G. Scott (2013). Epidemiological Modelling of Attention-Deficit/ Hyperactivity Disorder and Conduct Disorder for the Global Burden of Disease Study 2010. Journal of Child Psychology and Psychiatry, 54, pp. 1263-1274.

European Commission (2010). Special Eurobarometer: Science and Technology Report. [online] Available at: http://www.eceae.org/no/category/watching-brief/76/euro barometer-survey-shows-public-concern-on-animal-testing [Accessed 11 April 2017].

Fadali, M. (1996). Animal experimentation, a harvest of shame. Los Angeles, CA: Hidden Springs Press.

Gainetdinov, R.R. (2008). Dopamine Transporter Mutant Mice in Experimental Neuropharmacology. Naunyn-Schmiedeberg's Archives of Pharmacology, 377(4-6), pp. 301-313.

Garfield, E. and R.K. Merton (1979). Citation indexing: its theory and application in science, technology, and humanities, Vol. 8. New York: Wiley.

Greek, C.R. and J.S. Greek (2003). Sacred cows and golden geese, the human cost of experiments on animals. New York: Continuum. 
Hellsten, U., R.M. Harland, M.J. Gilchrist, D. Hendrix, J. Jurka, V. Kapitonov, I. Ovcharenko, N.H. Putnam, S. Shu, L. Taher, I.L. Blitz, B. Blumberg, D.S. Dichmann, I. Dubchak, E. Amaya, J.C. Detter, R. Fletcher, D.S. Gerhard, D. Goodstein, T. Graves, I.V. Grigoriev, J. Grimwood, T. Kawashima, E. Lindquist, S.M. Lucas, P.E. Mead, T. Mitros, H. Ogino, Y. Ohta, A.V. Poliakov, N. Pollet, J. Robert, A. Salamov, A.K. Sater, J. Schmutz, A. Terry, P.D. Vize, W.C. Warren, D. Wells, A. Wills, R.K. Wilson, L.B. Zimmerman, A.M. Zorn, R. Grainger, T. Grammer, M.K. Khokha, P.M. Richardson and D.S. Rokhsar (2010). The Genome of the Western Clawed Frog "Xenopus Tropicalis". Science, 328(5978), pp. 633-636.

Huhtala, A., L. Salminen, H. Tähti and H. Uusitalo (2008). Corneal Models for the Toxicity Testing of Drugs and Drug Releasing Materials. Topics in Multifunctional Biomaterials and Devices, 1(2), pp. 1-23.

Illman, J. (2008). Politics, protest, and progress: 10o years of animal research - The history of the research defense society. London: Research Defense Society.

Kilkenny, C., W. Browne, I.C. Cuthill, M. Emerson, and D.G. Altman (2010). Animal Research: Reporting In Vivo Experiments: The ARRIVE Guidelines. British Journal of Pharmacology, 16o(7), pp. 1577-1579.

Knight, A. (2007). The Poor Contribution of Chimpanzee Experiments to Biomedical Progress. Journal of Applied Animal Welfare Science, 10, pp. 281-308.

Lack, D. (1947). Darwin's finches. Cambridge: Cambridge University Press.

Lee, A.M., L.H. Tai, A. Zador and L. Wilbrecht (2015). Between the Primate and "Reptilian" Brain: Rodent Models Demonstrate the Role of Corticostriatal Circuits in Decision Making. Neuroscience, 296, pp. 66-74.

Long, X., J.Z. Huang and Y.S. Ho (2014). A Historical Review of Classic Articles in the Surgery Field. The American Journal of Surgery, 208(5), pp. 841-849.

Lucas, C., L.J. Criens-Poublon, C.T. Cockrell and R.J. de Haan (2002). Wound Healing in Cell Studies and Animal Model Experiments by Low Level Laser Therapy; Were Clinical Studies Justified? A Systematic Review. Lasers Medical Sciences, 17, pp. 110-134.

Macleod, M.R., T. O'Collins, L.L. Horky, D.W. Howells and G.A. Donnan (2005). Systematic Review and Meta-analysis of the Efficacy of FK506 in Experimental Stroke. Journal of Cerebral Blood Flow \& Metabolism, 25, pp. 1-9.

Martić-Kehl, M.I., J. Wernery, G. Folkers and P.A. Schubiger (2015). Quality of Animal Experiments in Anti-angiogenic Cancer Drug Development: - A Systematic Review. PLoS One, 10(9), pp. 1-14.

Nesse, R.M. and G.C. Williams (1996). Why we get sick: The new science of Darwinian medicine. New York: Times Books.

Parish, H. (1968). Victory with Vaccines. The story of immunization. Edinburgh: E. \& S. Livingstone. 
Pennisi, E. (1997). Monkey Virus DNA Found in Rare Human Cancers. Science, 275, pp. 748-749.

Perel, P., I. Roberts, E. Sena, P. Wheble, C. Briscoe, P. Sandercock, M. Macleod, L.E. Mignini, P. Jayaram and K.S. Khan (2007). Comparison of Treatment Effects Between Animal Experiments and Clinical Trials: Systematic Review. British Medical Journal, 334, pp. 197-206.

Perretta, G. (2009). Non-human Primate Models in Neuroscience Research. Scandinavian Journal of Laboratory Animal Science, 36(1), pp. 77-85.

Petropolis, D.B., D.M. Faust, M. Tolle, L. Rivière, T. Valentin, C. Neuveut, N. HernandezCuevas, A. Dufour, J.C. Olivo-Marin and N. Guillen (2016). Human Liver Infection in a Dish: Easy-to-build ${ }_{3} \mathrm{D}$ Liver Models for Studying Microbial Infection. PLoS One, 11(2), p. e0148667. [online] Available at: http://journals.plos.org/plosone/article? id=10.1371/journal.pone.0148667 [Accessed 11 August 2018].

Plotkin, S. (2017). Human Cell Strains in Vaccine Development. [online] Available at: https://www.historyofvaccines.org/content/articles/human-cell-strains-vaccine -development [Accessed 11 April 2017].

Pound, P., S. Ebrahim, P. Sandercock, M. Bracken and I. Roberts (2004). Where is the Evidence That Animal Research Benefits Humans?. British Medical Journal, 328, pp. 514-517.

Schaffner, A. (2006). Fieber-nützliches Oder Schädliches, zu Behandelndes Symptom? [Fever-useful or noxious symptom that should be treated?]. Therapeutische Umschau, 63(3), pp. 185-188.

Scheinin, L. and C.V. Wetli (2009). Sudden Death and Sickle Cell Trait: Medicolegal Considerations and Implications. The American Journal of Forensic Medicine and Pathology, 30(2), pp. 204-208.

Shapiro, K. (1998). Animal Models of Human Psychology: Critique of Science, Ethics, and Policy. Seattle: Hogrefe \& Huber.

Shively, C.A. and T.B. Clarkson (2009). The Unique Value of Primate Models in Translational Research. Nonhuman Primate Models of Women's Health: Introduction and Overview. American Journal Primatology, 71(9), pp. 715-721.

Viceconti, M., G. Clapworthy and S.V.S. Jan (2008). The Virtual Physiological Human. A European Initiative for In Silico Human Modelling. The Journal of Physiological Sciences, $5^{8}(7)$, pp. 441-446.

Viceconti, M., A. Henney and E. Morley-Fletcher (2016). In Silico Clinical Trials: How Computer Simulation Will Transform the Biomedical Industry. International Journal of Clinical Trials, 3(2), pp. 37-46.

Webber, B.J., D.J. Casa, A.I. Beutler, N.S. Nye, W.E. Trueblood and F.G. O'Connor (2016). Preventing Exertional Death in Military Trainees: Recommendations and Treatment Algorithms from a Multidisciplinary Working Group. Military Medicine, 181(4), pp. 311-318. 
World Health Organization (WHO) (2008). The Global Burden Of Disease: 2004 Update. Table A2. Burden of Disease in DALYs by Cause, Sex and Income Group in WHO Regions, Estimates for 2004 Geneva: World Health Organization.

Wilson, S.L., M. Ahearne and A. Hopkinson (2015). An Overview of Current Techniques for Ocular Toxicity Testing. Toxicology, 327, pp. 32-46. 